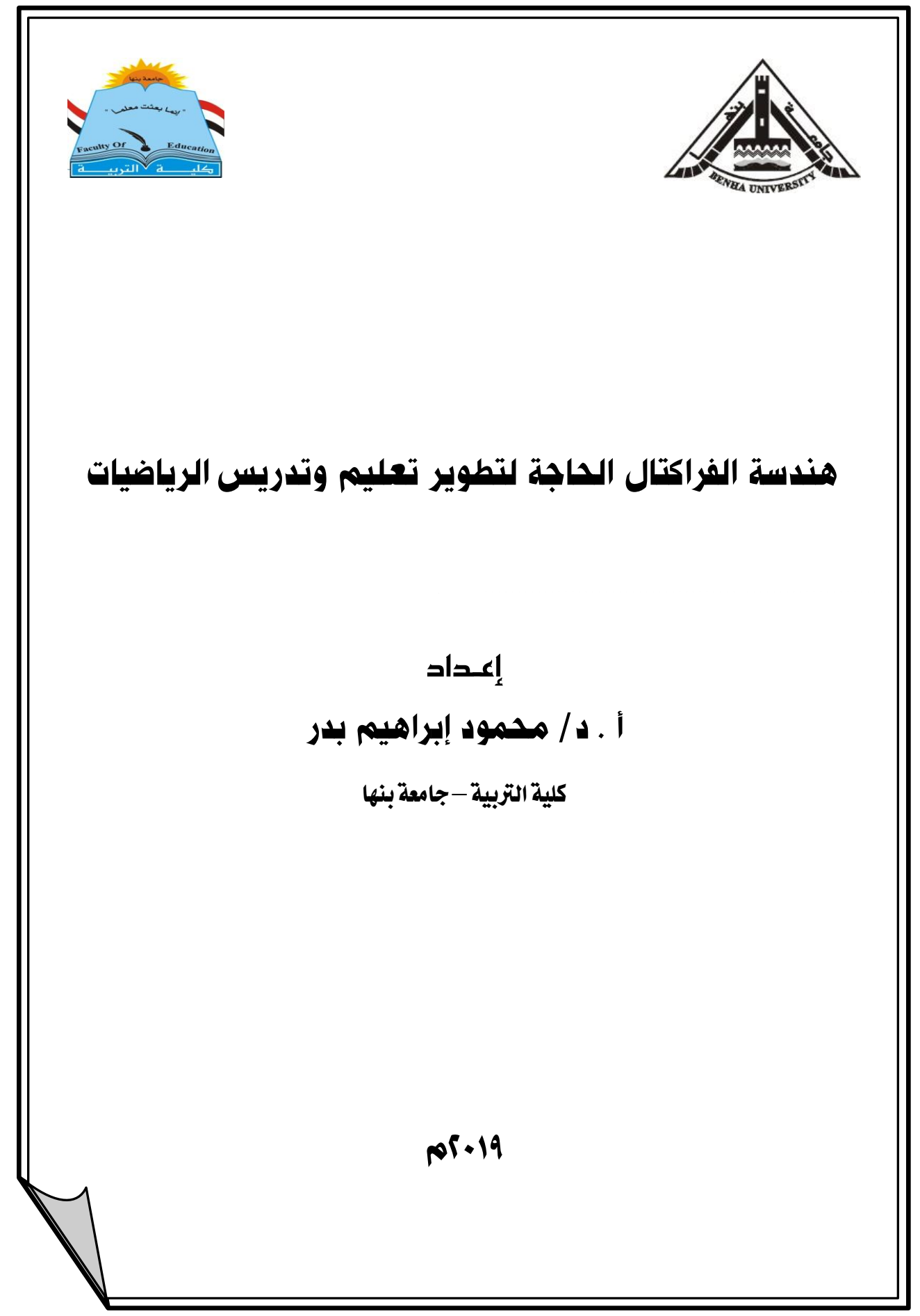




\section{هندسة الفراكتال الحاجة لتطوير تعليم وتدريس الرياضيات}

\section{إعـداد \\ أ أ ـ د / محمود إبراهيم بدر}

\section{كلية التربية - جامعة بنها}

بعد العصر الحالي عصر المعلومات والتقنية ، وقد شهدت بعض افرع الرياضيات تطور مذهل منذ اختراع

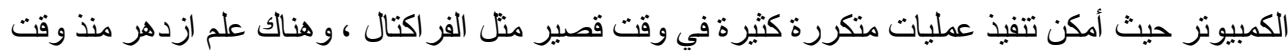

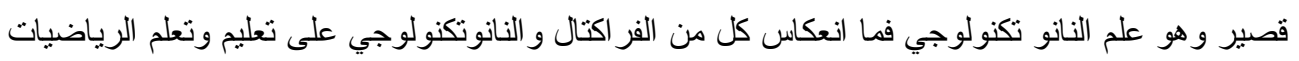
وما العلاقة بين النانوتكنولوجي و الفر اكتال:

\section{تعريف النانو تكنولوجي:}

فن استخدام ومر اقبة المو اد على مستوى مقاييس الأبعاد الذرية أو الجزيئية من أجل إنثاء الأجهزة المجهرية مثل الروبوت التي تستخدم في ذات المجال بنفس أبعاد دقائقه.

ويعرف أيضا بأنه علم تكنولوجيا هندسي بطبق عند أبعاد صغيرة جدا ، وعلم النانو

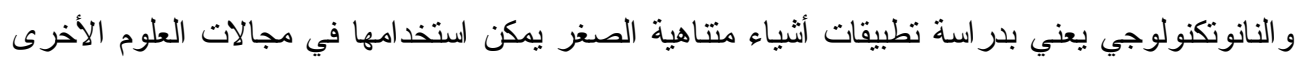

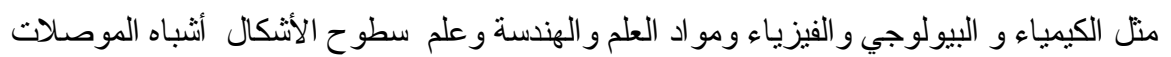

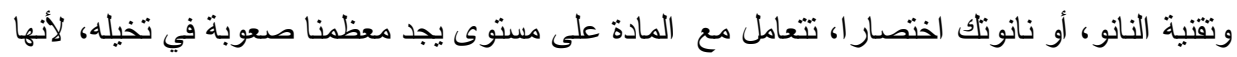

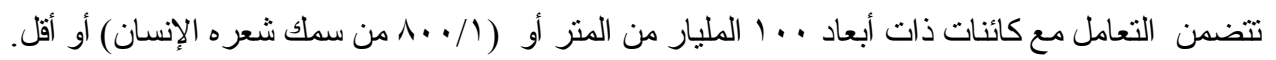
-

$$
\text { - }
$$

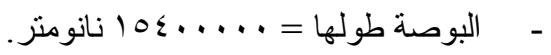

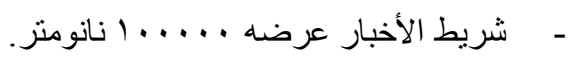

ويجادل العلماء في مستقبل نطبيقات النانوتكنولوجي حيث بستعون إلى ابتكار وتكوين مو اد و آلات جديدة

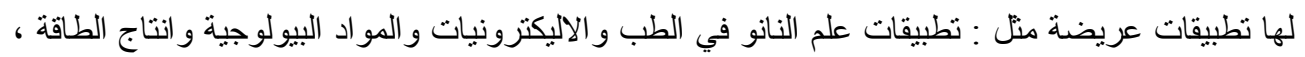
وفي ذات الوقت بثور جدل حول قضايا التلوث البيئي و اثر نلك المو اد النانونية و أثزها على الاقتصاد ومسنتقبل كفاية الغذاء للجنس البشري. 
و غالبأ ما تتغير الخصائص الكيميائية و الفيزيائية للمو اد إلى حد كبير عند نلك الأبعاد. وتستخدم

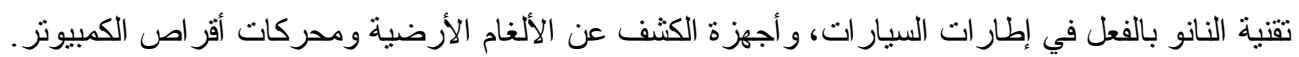
و الطب النانوي مجال: تخيل جسيمات في حجم خلية الدم التي يمكن إصدارها في في مجرى الأم على على شكل الروبوتات الصغيرة نسنطيع مهاجمة الخلايا السرطانية، أو "آلات" في في حجم الجزئ التئ يمكنها إصلاح الأضر ار الداخلية بالخلايا التالفة.

وفي المقياس النانوي، يمكن تغيير خصائص المواد. على سبيل المثال، و الصلابة، و التوصيل

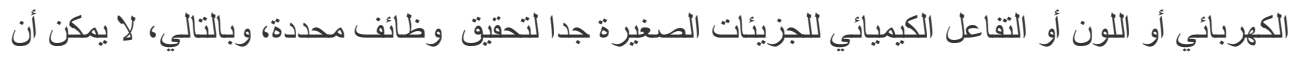

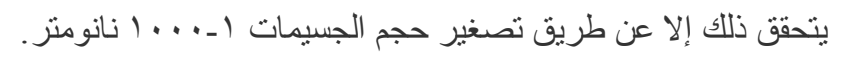
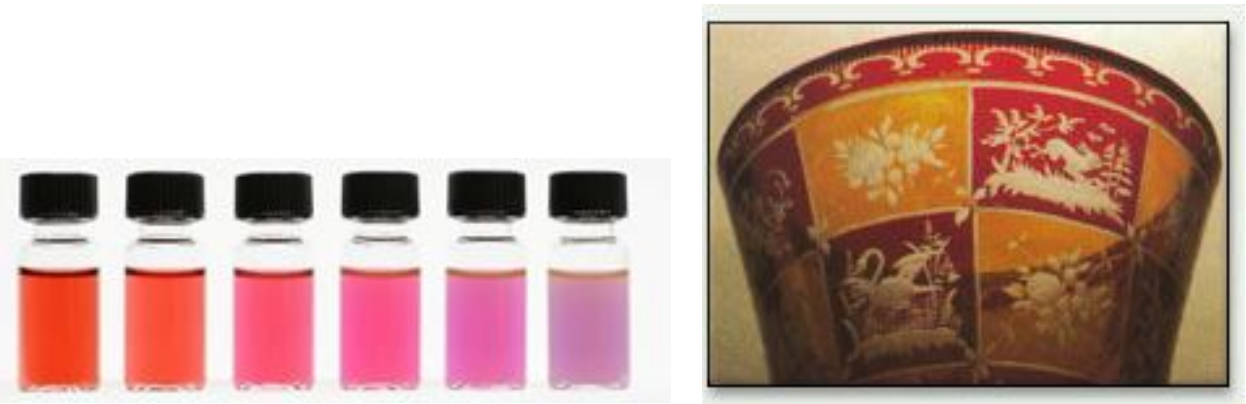


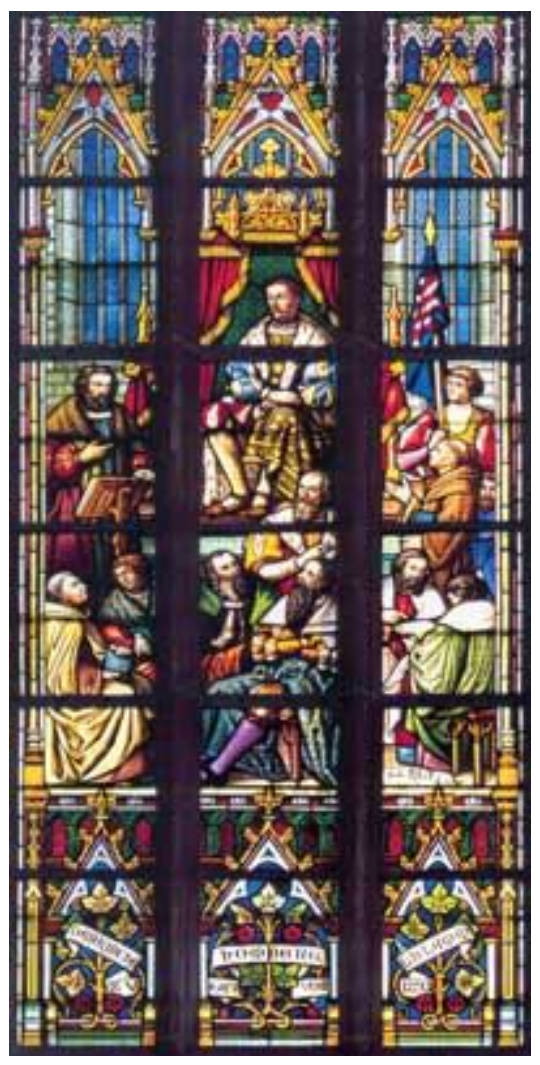

ونوجد نطبيقات معروفة لتكنولوجيا النانو ففي وقت سابق استخدم لون أحمر قاني تم استخدامه

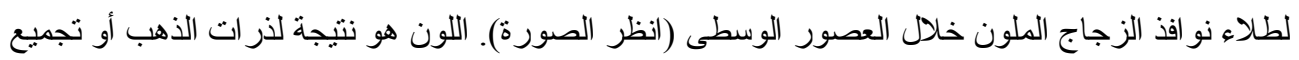

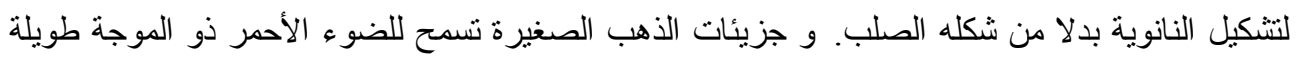

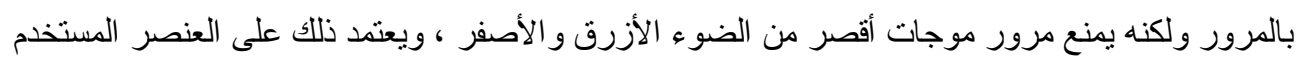

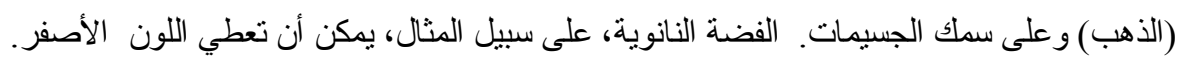

$$
\text { تغير لون الضوء المنتقل خلال الأهب النانوي }
$$

عندما يمر الضوء من خلال ورقة الذهب أقل من ... 1 نانومتر من ناحية السمك، نحصل على ضوء

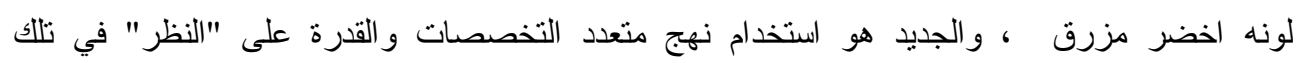

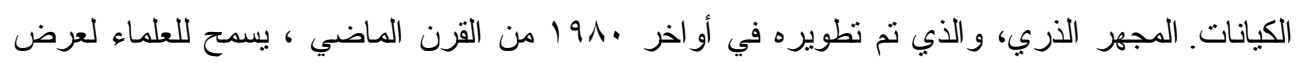

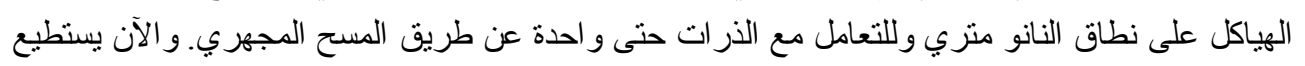




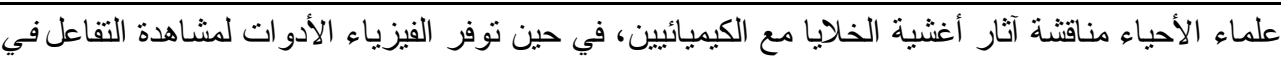

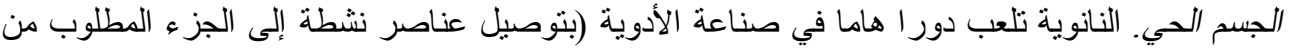

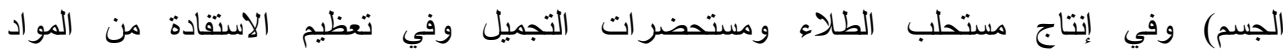
المحفزة. فتكنولوجيا النانو ، تجمع جميع العلوم الطبيعية وتخلق الرو ابط عبر بين التخصصات النتخلفة.

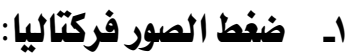

حفظ الصور بحجم صغير في الذاكرة بعني تقليص تكلفة الحفظ ووصول أسر ع للبيانات وهذا مفيد في كل من عمليات إنثارة التثغيل و الاتصال في الهندسة وهذا يفسر مواصلة الثركات و الجامعات لطريقة

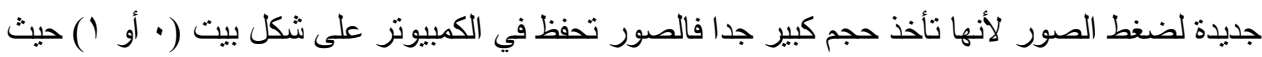

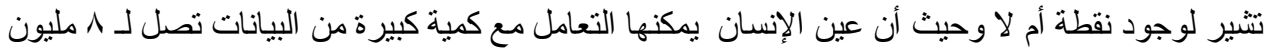

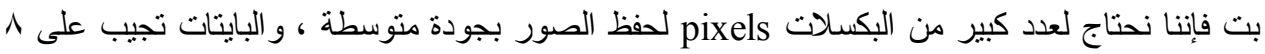
مليون تحدد الصورة و أكثر البيانات تحتوي كمية من الفو اصل التي يمكن حذفها عند الحفظ وتستبدل عند

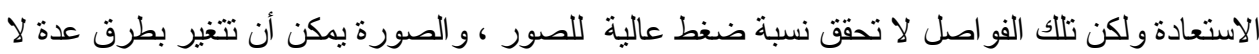

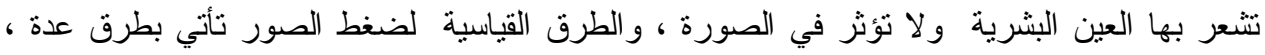

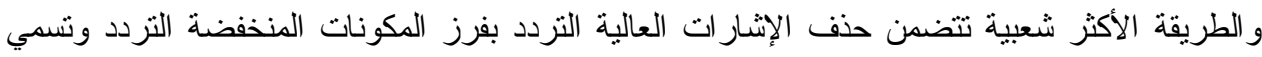

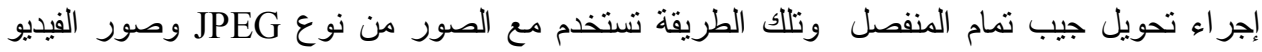

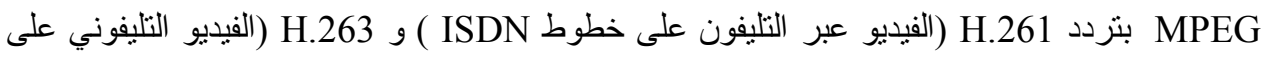
خطوط PSTN) ( ) ( )

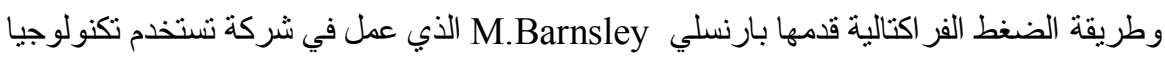

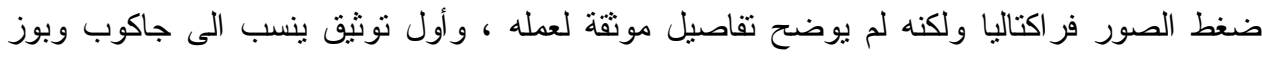
في مركز أنظمة نافل اوشن E.Jacobs and R.Boss سادييجو حيث استخدم تجزيء و تصنيف لقطع المنحني لكي يضغط المنحنيات عشو ائيا فر اكتاليا في بعدين و أول من نشر عن ضغط الصور فر فر اكتاليا هو طالب الدكتور اهب بارنسيلي .

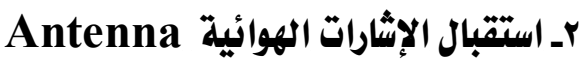

تتقيد الهنسة التقليدية بالموجات الطويلة ، وباستخدام خو اص الفر اكتال من المدكن الحصول على

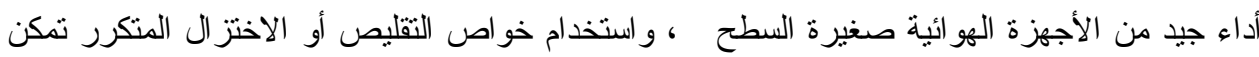

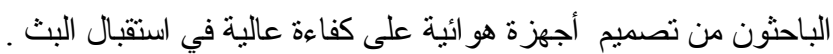


و الهو ائي الفراكتالي يبني مع نظرية تصميم الهوائي بالاستتاد على النظرية النقليدية

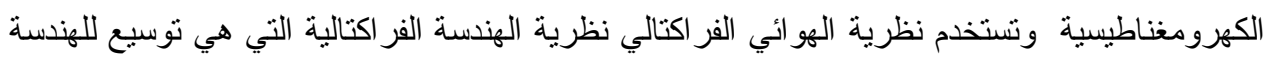

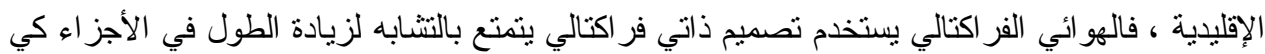

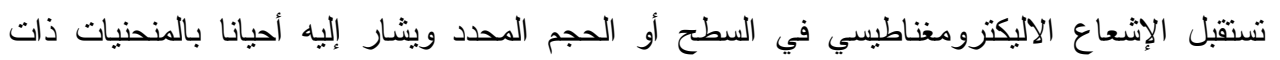

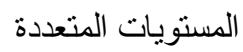

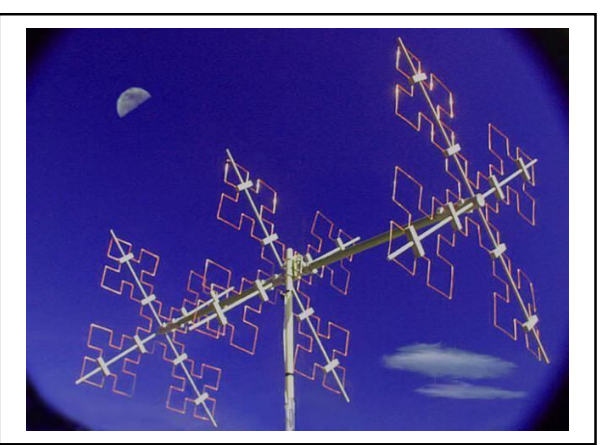

و لهذا السبب تعد الهو ائيات الفر اكتالية لها تطبيقات في في الهو اتف الخلوية و الاتصالات باستخدام الموجات القصيرة جداب

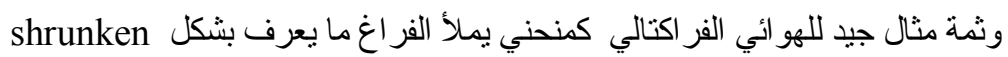
حيث يكون سلك النحاس صغير ومقارب للطول الموجي ويتميز الهو ائي الفر اكتالي بالأداء المثناز لكل النزرددات

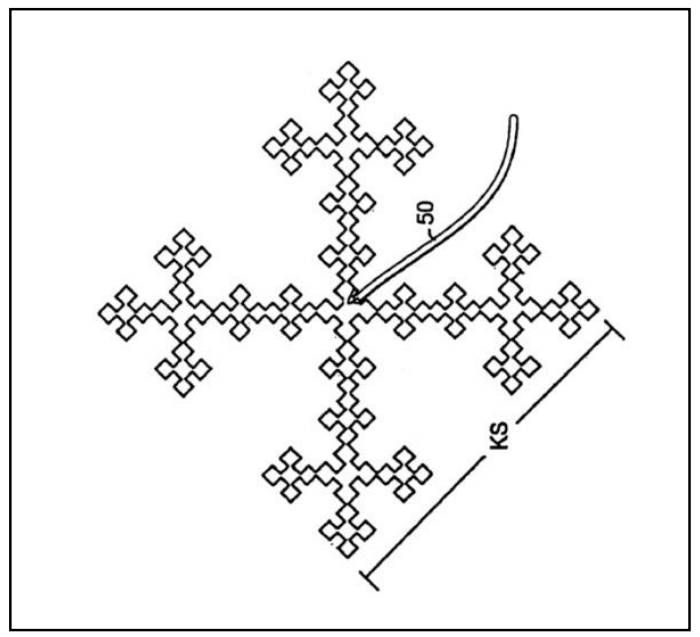


هو ائي فر اكتالي : منحني يملأ الفراغ يسمي Shrunken helix

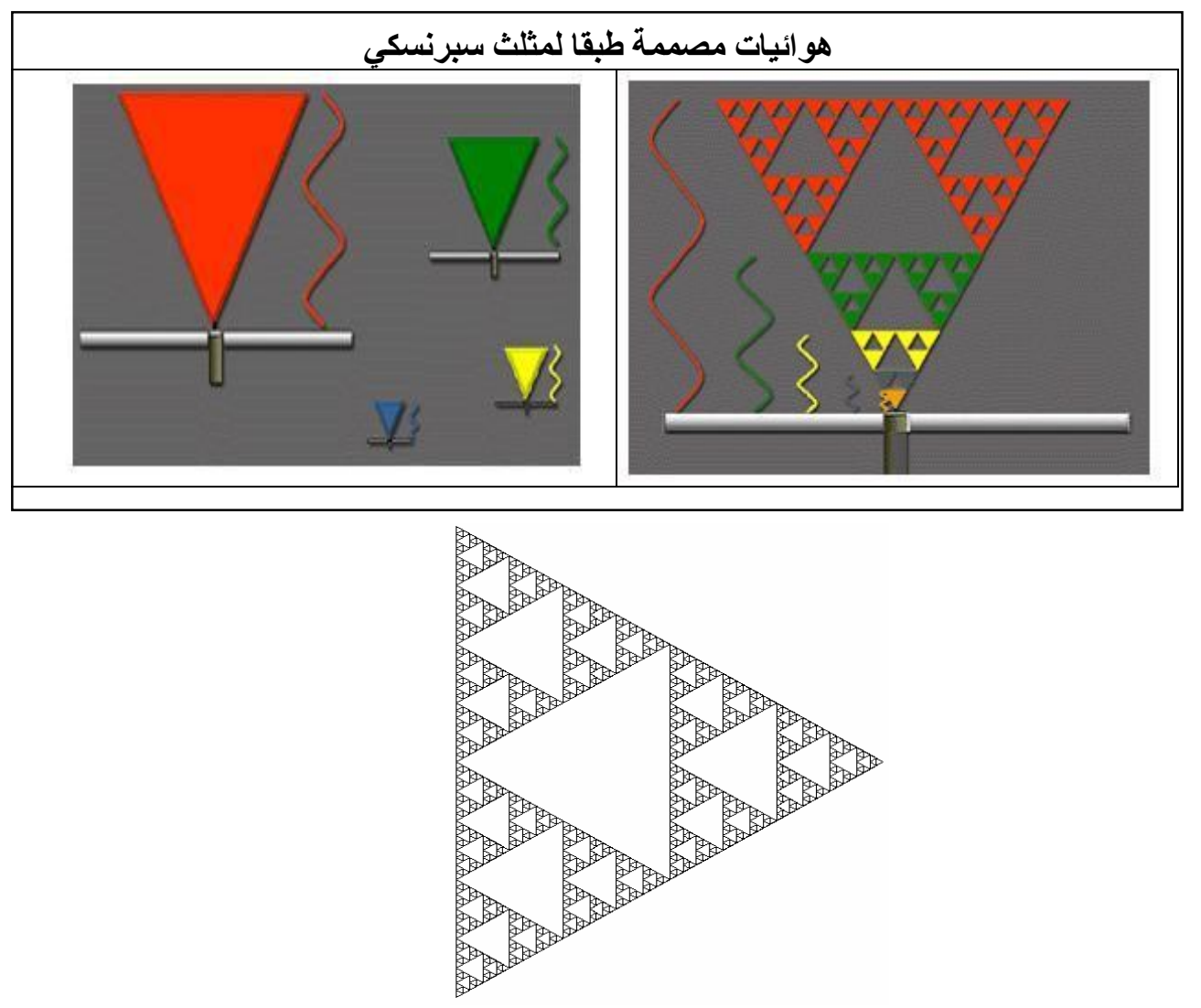

to Allgasket

; note - draws 7 stages one after the other

cs gasket 10241024

gasket1 1024512

gasket1 1024256

gasket1 1024128

gasket 1102464

gasket1 102432 
gasket1 102416

ht

end

to gasket :x :n

;note - clears screen, positions cursor, draws gasket size $\mathrm{x}$, smallest triangle size y

cs pu bk 30 lt 90 fd 200 rt 90 pd

gasket $1: \mathrm{x}: \mathrm{n}$

end

to gasket $1: \mathrm{x}: \mathrm{n}$

; note - draws gasket size $\mathrm{x}$, smallest triangle size $\mathrm{y}$, from current position if : $\mathrm{x}<: \mathrm{n}$ [stop]

repeat 3 [gasket1 :x/2 :n fd :x/2 rt 120]

end

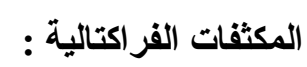

converters وتستخدم بكثرة في تحويل البيانات IC المكثفات واحدة من الدوائر المتكاملة :

وذبذبات الراديو RF وتستخدم الأثكال الهندية الفر اكتالية التي يمكن بنائها في شكل عمليات

رقمية قياسية

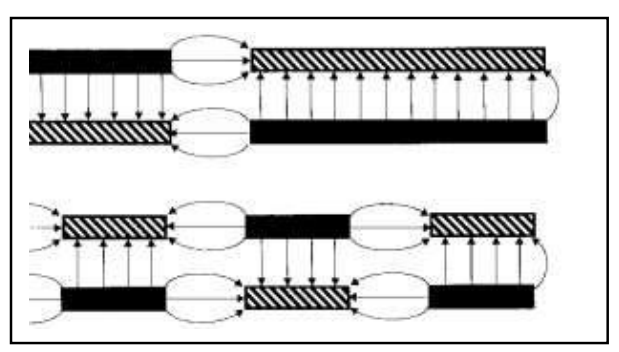


مجلة كلية التربية ببنها

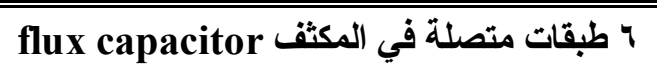

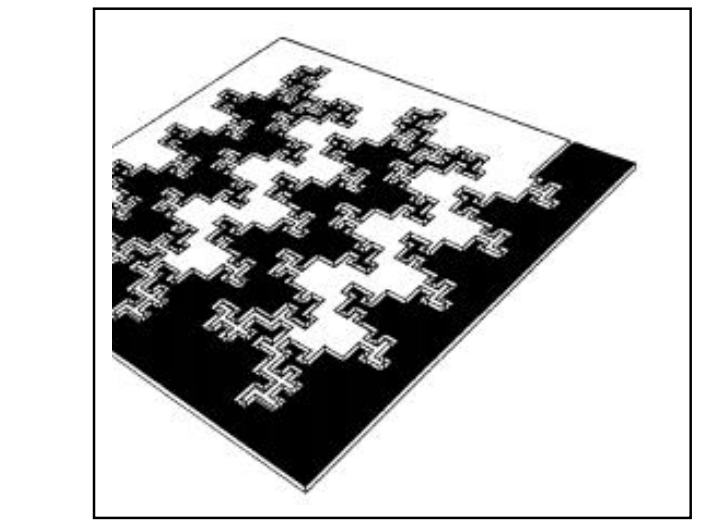

مكثف فر اكتالي ثلاثي الأبعاد يستخدم طبقة معدنية واحدة

الفر اكتالات ذات الأبعاد الكبيرة تؤدي لمكثفات كبيرة السعة

Fractal Filters, Resonators and مرشحات الصدي والمقاومة الفر اكتالية الماتية

Resistors

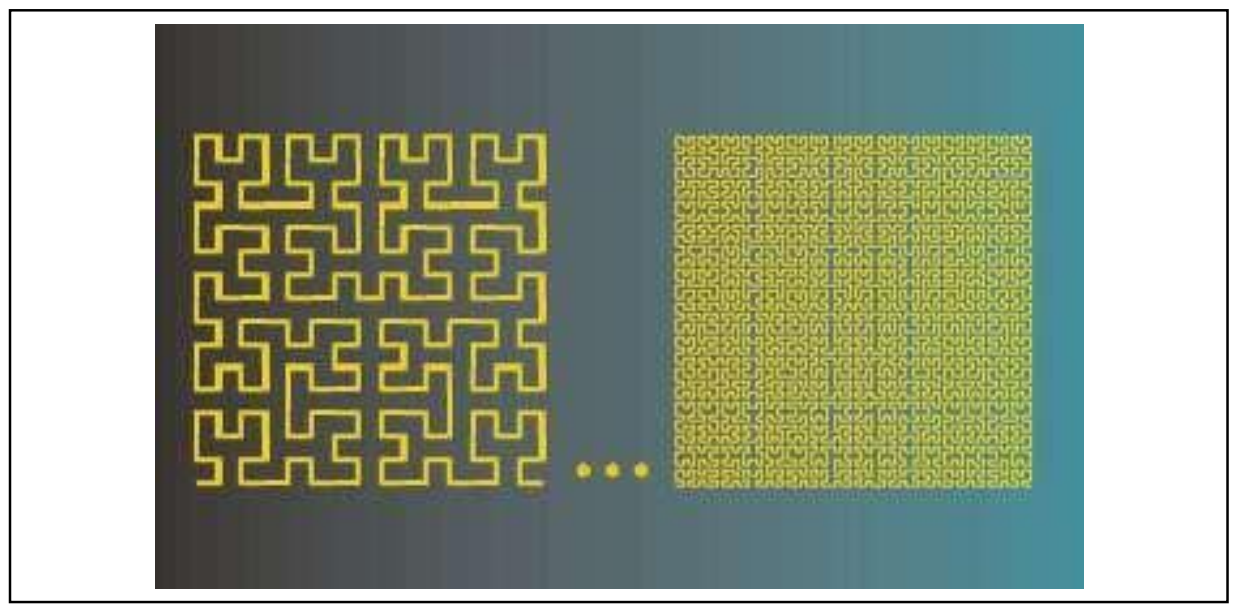




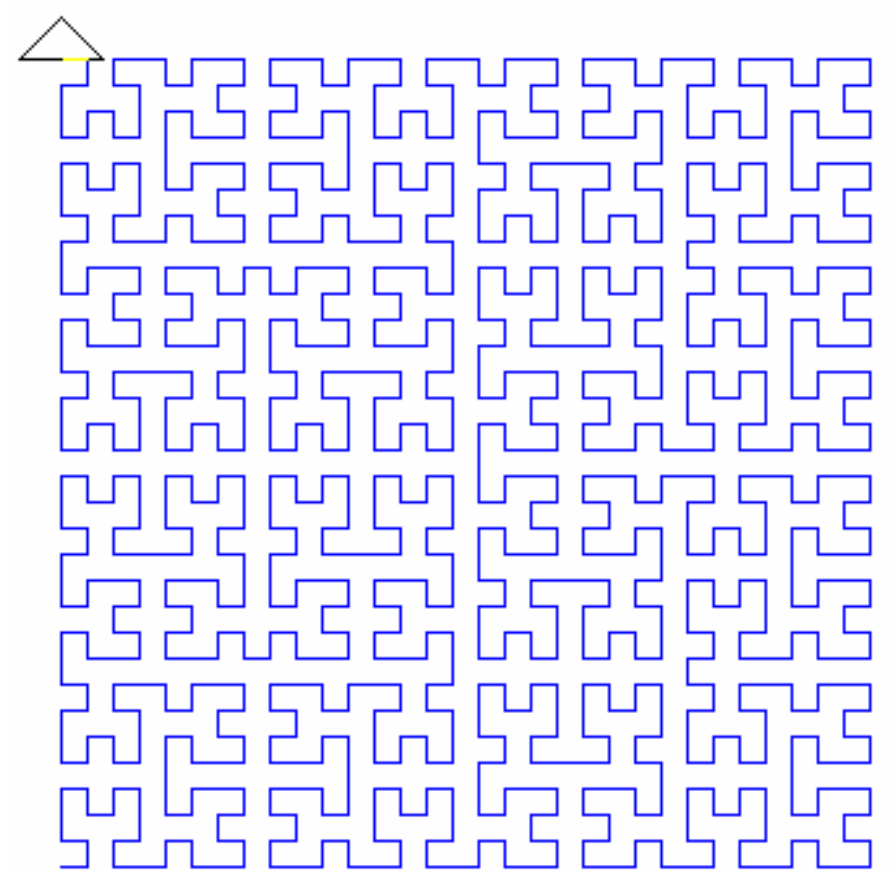

TO Hilb $: \mathrm{n}: \mathrm{a}: \mathrm{h}$

IF $: n=0[$ STOP ]

RT :a Hilb :n - 1 (-:a) :h FD :h LT :a Hilb :n - 1 :a :h FD :h

Hilb :n - 1 :a :h LT :a FD :h Hilb :n - 1 (-:a) :h RT :a

END

TO Hilbert

PU SETPOS [-150 -150] PD SETPC [000 000 255]

Hilb 59010

END 


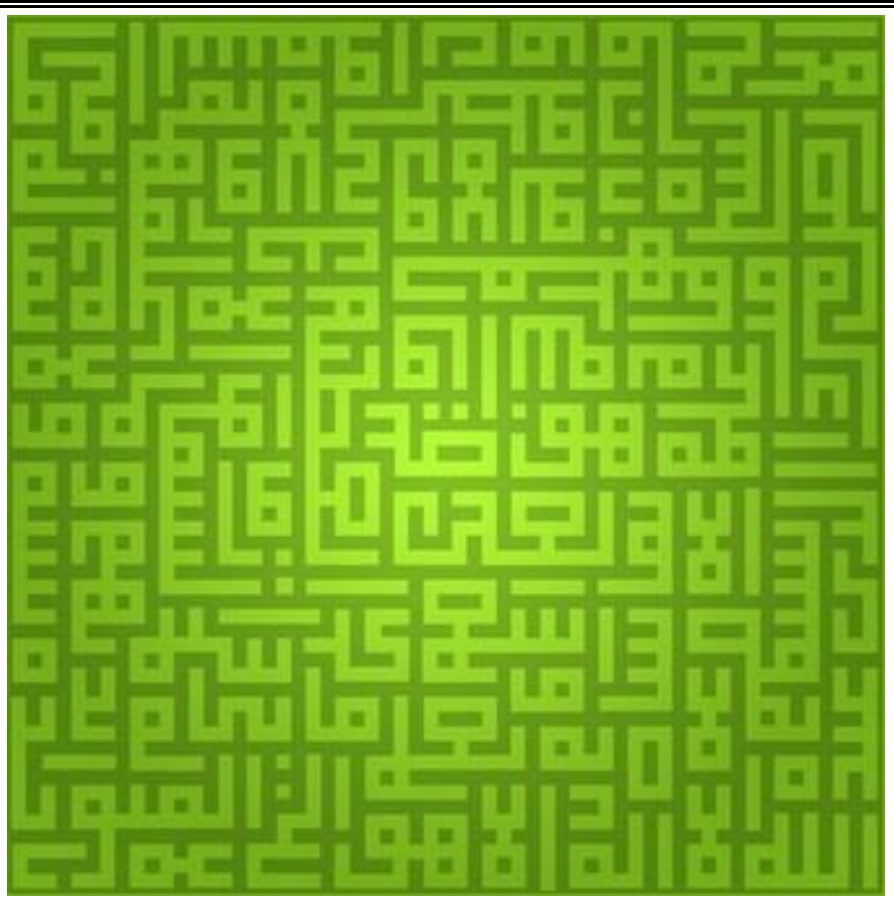

سورة الكرسي من القرآن الكريم

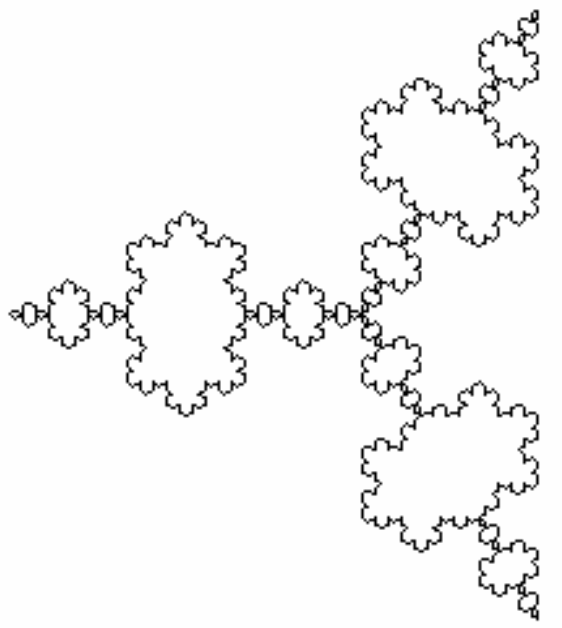

cs fractal $35700 \mathrm{ht}$ 


\section{to DrawFractalLinea :level :length :n}

\section{ifelse :level $<1$ [}

forward :length] [

DrawFractalLine (sum -1 :level) (quotient :length :n) :n

rt (360/:n )]

end

to fractal :n :level :length

repeat :n [ DrawFractalLinea :level :length :n rt (360 /:n)]

end

\section{تطبيقات هندسة الفراكتال في النانوتكنولوجي:}

'التجميع الذاتي :' بحمل نطور و اعد كأسلوب لبناء نانو -الدوائر التجارية. واستخدام هذا النهج، سمح

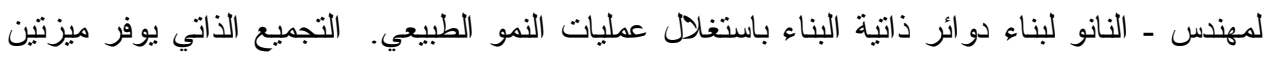

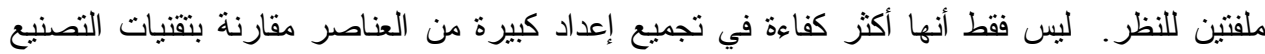

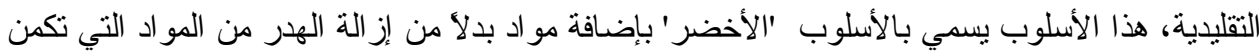

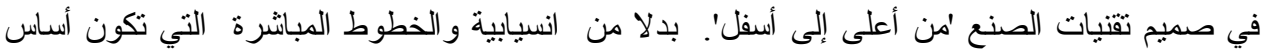
تصميم الدو ائر الاليكترونية اليوم .

و عمليات التجميع الذاتي تتشئ نماذج من هندسة الفر اكتال ، و هي أثكال تتكرر بشكل مشابه وهي أنثكال

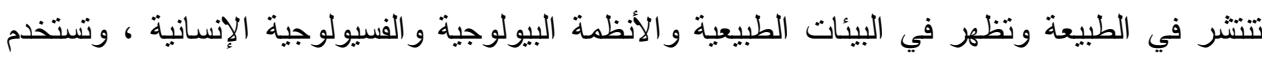

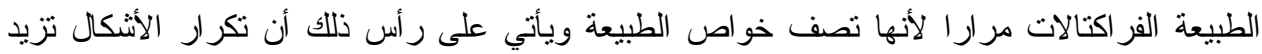

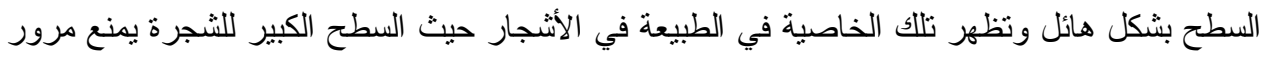

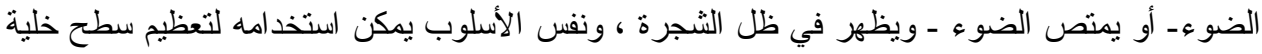

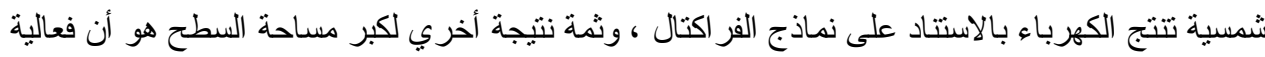

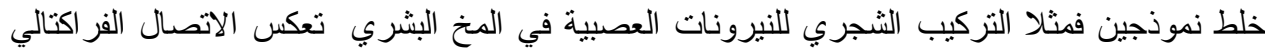

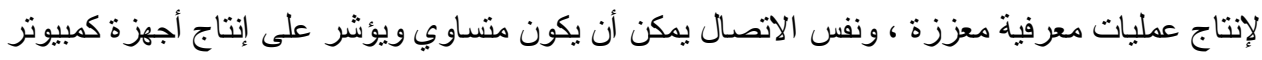

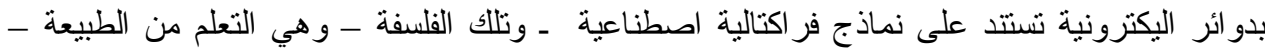

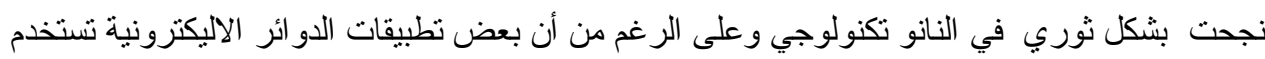

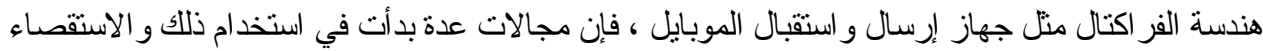

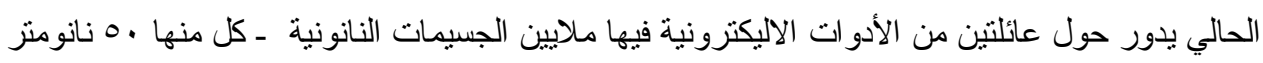

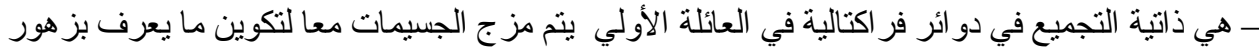

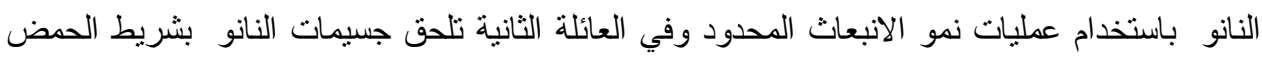


النووي الذي يجمع لتكوين دائرة فركتالية وكلتا الحالتين تتشأ عمليات نولا نموذج فركتالي شبيه بالثجرة.

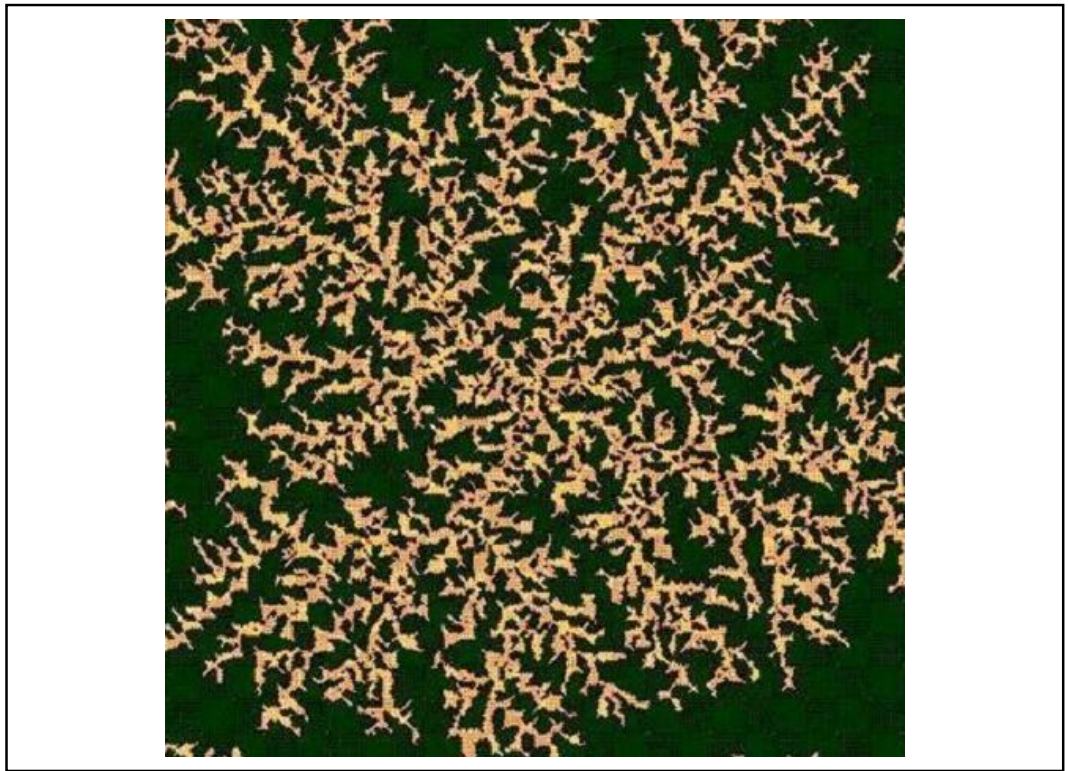

شكل يوضح محاكاة تجميع دوائر اليكترونية فر اكتالية

وتللك يقود شروط نطور ها الخصائص الفر اكتالية للدو ائر مشابه لنظيرتها الطبيعية في المخ البشري ، ونتخيل في المستقبل أن يعمل الكمبيوتز بطريقة مشابهة للمخ حيث تعمل الدو ائر الفركتالية كنسيج مدر ج في منطقة معينة من الدماغ يحسن أو يحفظ وظائف المريض الذهنية

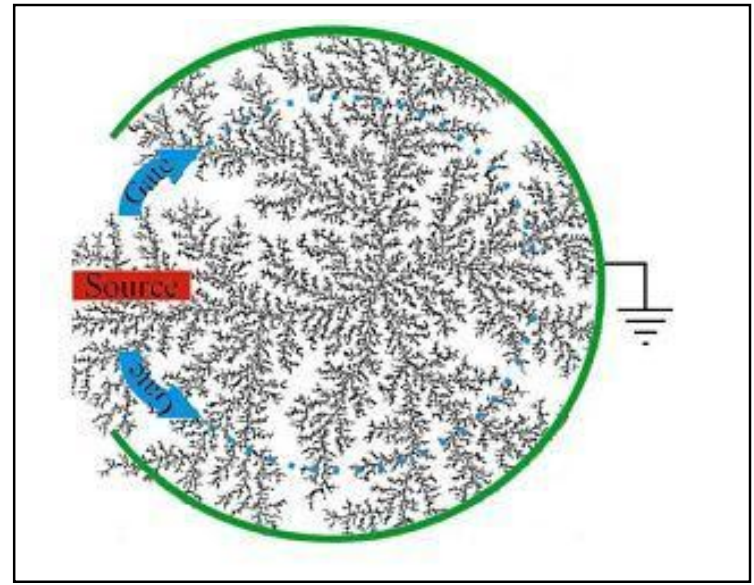




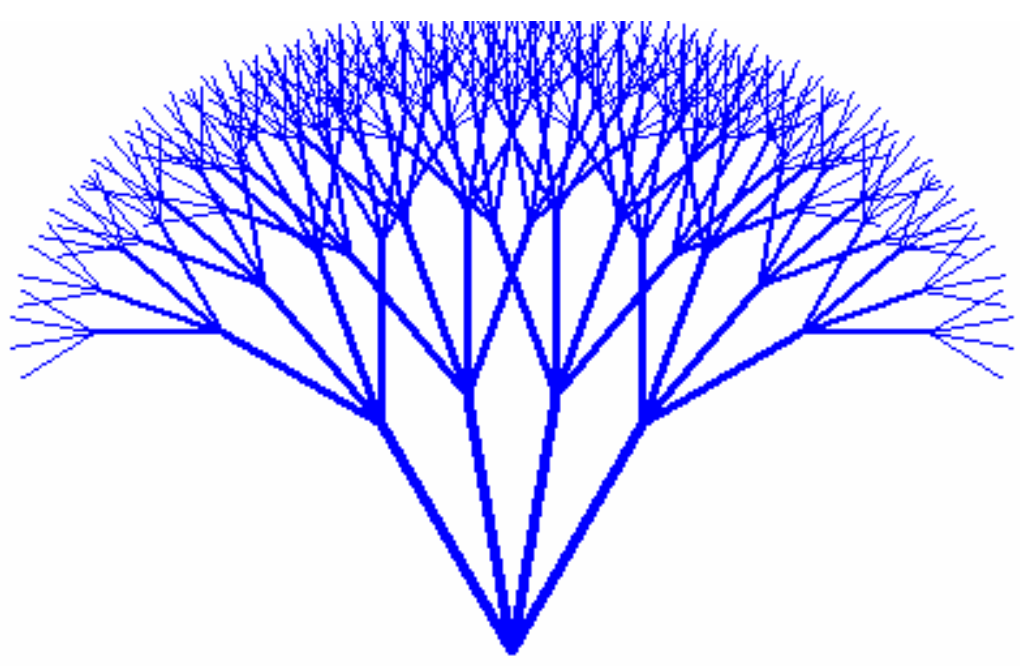

cs ht tree 60100340.7

to tree :angle :size :level :branches :ratio

localmake "x0 first pos

localmake "y0 first butfirst pos

localmake "th heading

make "branch :angle/(:branches-1)

repeat :branches [

setpensize list :level+1 :level+1

$\mathrm{pu}$

setxy : 0 : $\mathrm{y} 0$

setheading :th-:angle/2+:branch*(repcount-1)

pd

fd :size

if :level $>0$ [ tree :angle :size*:ratio :level-1 :branches :ratio]

]

end 
شكل يوضح تأثير مجال الفراكتال على الموصلات

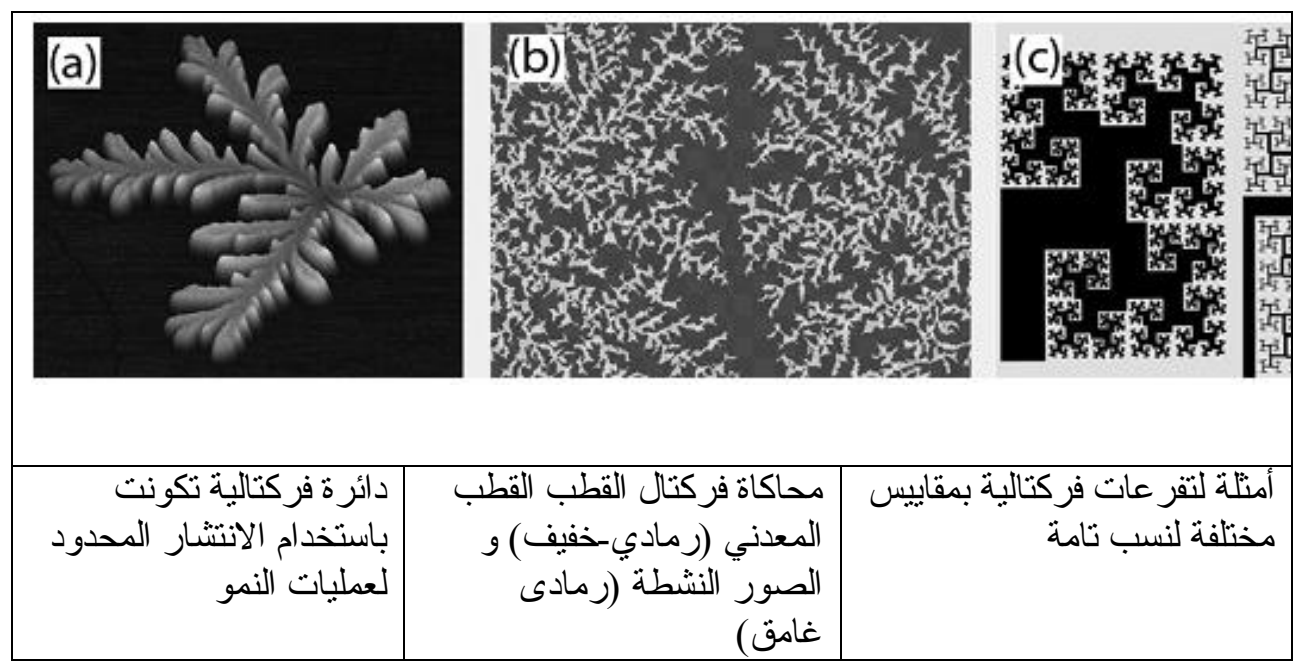

وتوجد تطبيقات أخري لهندسة الفر اكتال في إثار ات الكلام و أنظمة تمييز الصوت حيث تحظي

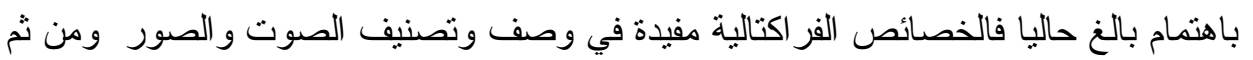
فالفر اكتالات تقدم وصف مبسط لكثير من الأشياء الطبيعية .

و أخير ا يهنم علم النانوي بالبحث عن وحدات صغيرة تشابه أشياء كبيرة و هذا بشدة ببعض مفاهيم الرياضيات منل التوازي وتتليث الأضلاع و الزو ايا و التشابه وقوانين الأسس التساه

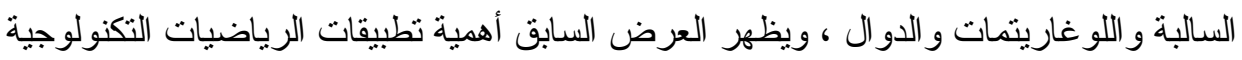

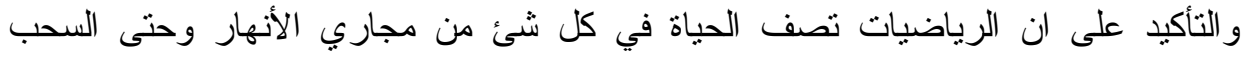

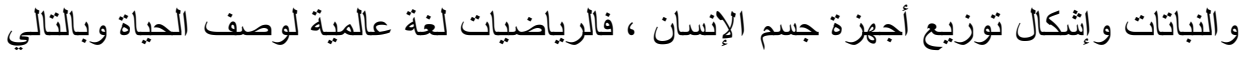

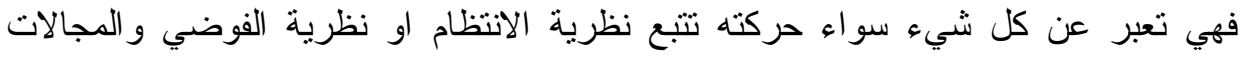

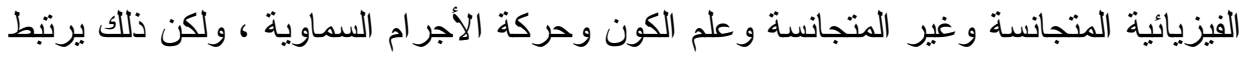
بطريقة تقديم الموضوعات و محتو اها ومناسبته للمرحلة العمرية ، إذ ينبغي مر اعاة المستوي

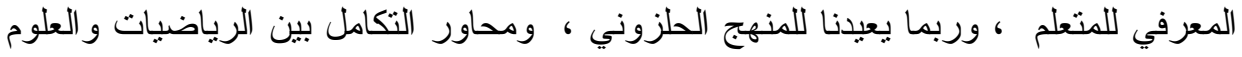

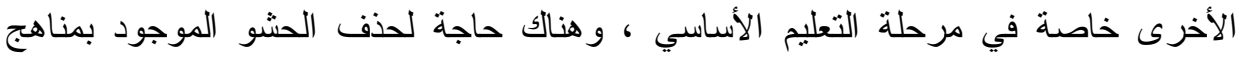

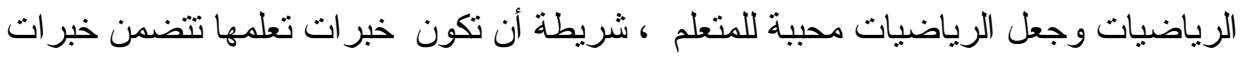




$$
\text { سارة منل النجاح و الفهم و التقهم لدور ها. }
$$

فالرياضيات لها تطبيقات في الفيزياء و التكنولوجيا و الكيمياء و البيولوجي و الجيولوجيا

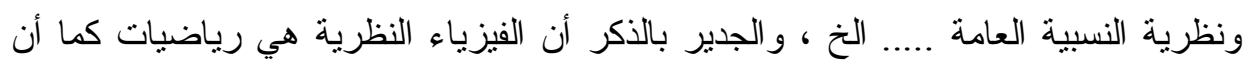

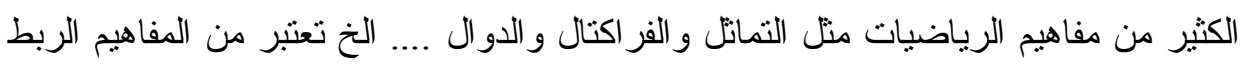
ليس بين فروع الرياضيات فقط بل بين الرياضيات و العلوم الأخرى. لتدريس الفر اكتال هناك حاجة لنطوير مقررات الرياضيات :

اـ تدريس لغة اللوجو عبر سنوات دراسية عديدة فهي تساعد على فهم كيفية تصميم

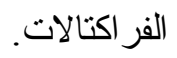

$$
\text { r ـ ت تدريس مفاهيم الز اوية و التتصيف و التو ازي و التتليث للأضلاع و الزو ايا و المضلعات . }
$$

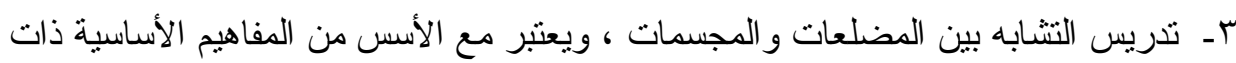

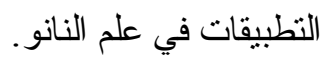

عـ ـ لتدريس الفر اكتال الجبري يجب تدريس مفاهيم : الأعداد المركبة و رسم الدوال و الاستعانة

$$
\text { بيرمجيات جاهزة مثل : XaoS. }
$$

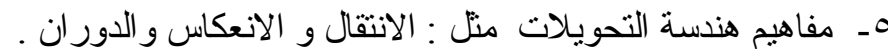

$$
\begin{aligned}
& \text { 7ـ - الربط بين الفر اكتال و الطبيعة و التكنولوجيا و التمانل. }
\end{aligned}
$$

V- مقدمة عن نظرية الفوضي ويحسن ان تكون في المرحلة الثانوية. ^ـ ـ قو انين الأسس عند تقديم الفر اكتالات المتقدمة مثل الجبرية و اللو غارتمات للبعد الفر اكتالي.

$$
\text { 9- يمكن تدريس رسم الفر اكتالات يدويا في : }
$$

- الصفوف العليا من المرحلة الابتدائية وبطريقة استكثافية بالتز امن مع مفاهيم

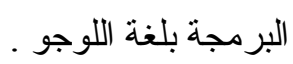

- وفي المرحلة الإعدادية يمكن تدريس خصائص الفر اكتالات ، ورسمها بلغة

$$
\text { اللوجو . مافي }
$$

- أما المرحلة الثانوية فيمكن تدريس الفر اكتال الجبري ونطبيقات الفركتالات في

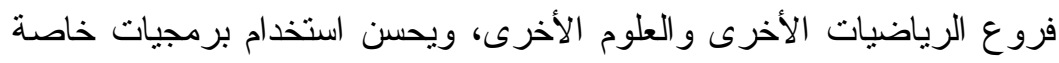

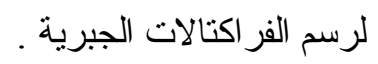




\section{المراجـ}

- Vladimir Batagelj, DRAWING SPACE-FILLING CURVES IN LOGO,

- Fractal Pack 1 , Educators' Guide, Fractals Are SMART: Science, Math \& Art!, MSWLOGO,G. Mills ,ver. 6.4h modified Arabic version by Mahmoud Badr,2002, http://softronics.com

- أحمد حسين حسن ، "فاعلية برنامج مقترح قائم على التطبيقات الرياضية لهندسة

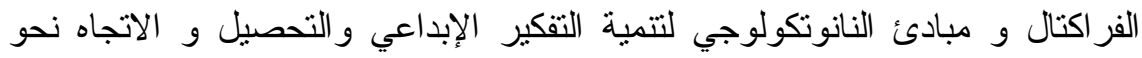
الرياضيات لاى طلاب المرحلة الإعدادية" ، رسالة دكتور اه غير منشورة ، كلية البنات

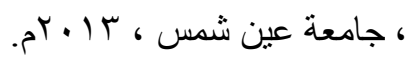

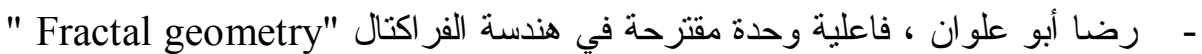
لطلاب الرياضيات بكلية التربية ، الجمعية المصرية للمناهج وطرق التدريس ، مجلة الندان

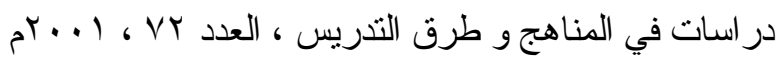
- رضا أبو علوان ، تضمين هندسة الفراكتال "Fractal geometry " في الرياضيات

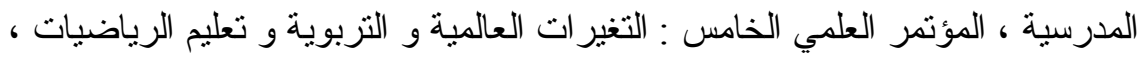

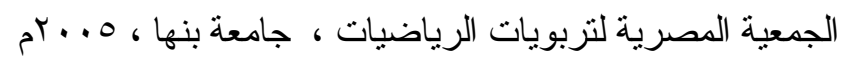
- محد فخري العشري ، فاعلية برنامج تعلم اليكتروني مدمج في تدريس هندسة الفر اكتال

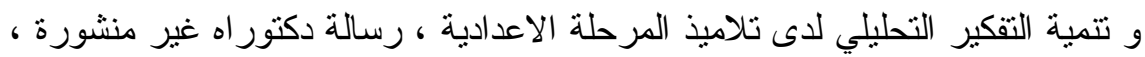

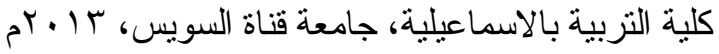

